\title{
THE DEGREE OF A LINEAR HOMOGENEOUS GROUP
}

\author{
BY \\ WILLIAM BENJAMIN FITE
}

The problem considered in this article is that of the relation between the degree of a linear homogeneous group and the abstract properties of the group. We have the theorem of Frobenius $\left({ }^{1}\right)$ to the effect that the degree of an irreducible group is a divisor of the order of the group, and then the generalization of this by Schur $\left({ }^{2}\right)$ which states that the order of the group is divisible by the product of the degree and the order of the central. In these Transactions $\left(^{3}\right)$ I called attention to the importance of the commutators of the group in this connection by showing that the degree of an irreducible group is not less than the order of any invariant commutator. As a matter of fact, it is a multiple of this order. It is shown in the present paper that the orders of certain non-invariant commutators also have a bearing on the degree of a linear group, whether it be reducible or irreducible. Moreover for groups of a certain category the degree is a multiple of the order of the whole commutator subgroup.

If a linear homogeneous group has a similarity substitution as a commutator, the degree of the group is a multiple of the order of this commutator since all the multipliers of a similarity substitution are equal and the determinant of a commutator is equal to one. If $G$ is an irreducible group of order $g$ and degree $n$ with a central of order $\alpha$ all its invariant substitutions are similarity substitutions, and if $\beta$ is the order of an invariant commutator $\beta^{2}$ is a divisor of $g$ since $g$ is divisible by $n \alpha$ and $\alpha$ and $n$ are both divisible by $\beta$. If $G$ is reducible at least one of its irreducible components has an invariant commutator whose order is equal to the highest order of any multiplier of any invariant commutator of $G$. Therefore the degree of this component is a multiple of this order, and the degree of $G$ is greater than this order. Hence we have the following theorem.

THEOREM I. The degree of a linear group is not less than the order of any multiplier of any invariant commutator of the group, and if the degree is equal to this order the group is irreducible.

It is not necessary that the commutator mentioned in the theorem be invariant in the group. For if

Presented to the Society, April 29, 1943; received by the editors October 27, 1943.

(1) Frobenius, Preuss. Akad. Wiss. Sitzungsber. (1896) $\$ 12$.

(2) Schur, J. Reine Angew. Math. vol. 127 (1904) p. 44.

(3) Fite, Trans. Amer. Math. Soc. vol. 7 (1906) p. 66. 


$$
A^{-1} T A=T T_{1}
$$

where $T_{1}$ is commutative with $A$, we have

$$
A^{-x} T A^{x}=T T_{1}^{x}
$$

If now $T$ and $T_{1}$ are commutative both can be assumed to be in normal form. Then if $\omega$ is a multiplier of $T_{1}$ of $\operatorname{order} \beta$ and $\rho$ is the corresponding multiplier of $T, \rho \omega^{x}$, where $x=1,2, \cdots, \beta$, are distinct multipliers of $T$. Hence the degree of $G$ is at least $\beta$.

Theorem II. The degree of a linear group $G$ of order $p^{m}$ ( $p$ an odd prime) is not less than $p^{\beta}$, where $p^{\beta}$ is the order of a commutator $T_{1}$ such that

and

$$
A^{-1} T A=T T_{1}
$$

$$
A^{-1} T_{1} A=T_{1} T_{2},
$$

$T$ being commutative with $T_{1}$ and $T_{2}$ being invariant in $G$.

The order of $T_{2}$ does not exceed $p^{\beta}$. If it equals $p^{\beta}$ the conclusion follows from Theorem I. Suppose then that $T_{2}$ is of order less than $p^{\beta}$. We can assume that $T, T_{1}$, and $T_{2}$ are all in normal form since they are commutative. Then if $\omega$ is a multiplier of $T_{1}$ of order $p^{\beta}$, the multiplier of $T_{2}$ that corresponds to $\omega$ is of the form $\omega^{j p \gamma}$ where $j \neq 0(\bmod p)$ and $0<\gamma \leqq \beta$. Now

$$
A^{-x} T A^{x}=T T_{1}^{x} T_{2}^{x(x-1) / 2}
$$

If $\rho$ is the multiplier of $T$ corresponding to $\omega$ of $T_{1}$ then

$$
\rho \omega^{x+j p^{\gamma} x(x-1) / 2}
$$

is a multiplier of $T$. These are all distinct for $x$ less than $p^{\beta}$, for if

then

$$
x+\frac{j p^{\gamma} x(x-1)}{2} \equiv y+\frac{j p^{\gamma} y(y-1)}{2}\left(\bmod p^{\beta}\right)
$$

or

$$
2(x-y)+j p^{\gamma}\left(x^{2}-y^{2}\right)-j p^{\gamma}(x-y) \equiv 0,
$$

$$
2 s+j s p^{\gamma}(x+y)-j s p^{\gamma} \equiv 0\left(\bmod p^{\beta-\delta}\right),
$$

where $p^{\delta}$ is the highest power of $p$ that divides $x-y$, and $s \neq 0(\bmod p)$. But this is impossible since $\delta<\beta$.

Let $G$ be an irreducible group of degree $n$ and let $T$ be a substitution of $G$ that corresponds to an invariant operation of the central quotient group $G^{\prime}$ of the highest order, say $\beta(\beta>1)$. Then $T$ is invariant in a subgroup $G_{1}$ of index $\beta$, since it has exactly $\beta$ conjugates. Moreover $T$ is commutative with 
every commutator of $\left.{ }^{4}\right) G$ and therefore $G_{1}$ is invariant in $G$, the quotient group $G / G_{1}$ being cyclic of order $\beta$. The substitution $T$ and the central $H$ of $G$ generate an invariant abelian subgroup of $G$ not all of whose substitutions are similarity substitutions. It then follows from a theorem of Blichfeldt $\left(^{5}\right)$ that $G$ is imprimitive, each system of imprimitivity consisting of those variables that have the same multiplier in $T$. Among these multipliers of $T$ there are at least $\beta$ distinct ones. Hence there are at least $\beta$ systems of imprimitivity. The variables of each system are transformed among themselves by every substitution of $G_{1}$ and the systems are permuted among themselves by the substitutions of $G$ in accordance with a transitive cyclic permutation group of order $\beta$. Hence there are $\beta$ distinct multipliers of $T$, each occurring $n / \beta$ times.

The substitutions of $G_{1}$ as far as they affect the variables of any one system form an irreducible group, since the variables of any one system are transformed into linear functions of the variables of another system by any substitution of $G$ not in $G_{1}$. These irreducible groups, one in each system and each of degree $n / \beta$, are irreducible components of $G_{1}$. They are simply isomorphic since they are conjugate.

If $\beta_{1}$ is the highest order of any multiplier of an invariant commutator of $G_{1}$ there is an invariant commutator of one of the irreducible components of $G_{1}$ of order $\beta_{1}$. The degree of this component, and therefore that of every one of these irreducible components of $G_{1}$, is a multiple of $\beta_{1}$. Hence $n$ is a multiple of $\beta \beta_{1}$.

If $n=\beta$ the irreducible components of $G_{1}$ are all of degree 1 , and therefore abelian. Hence $G_{1}$ is abelian. Conversely, if $G_{1}$ is abelian $n=\beta$. Hence we have the following theorem.

Theorem III. A necessary and sufficient condition that $G$ be of degree $\beta$ is that $G_{1}$ be abelian.

This means, among other things, that if $G$ is simply isomorphic with an irreducible group of degree $\beta$ it is not simply isomorphic with an irreducible group of any other degree. It is also an immediate consequence of the theorem that if $G$ has an invariant commutator of order greater than $\beta$ then $G_{1}$ is not abelian, for in this case $n>\beta$.

If $n=\beta$ the multipliers of $T$ are all distinct. Then each system of imprimitivity of $G$ contains only one letter. Hence $\left(^{6}\right)$ we have:

TheOREM IV. An irreducible group whose degree is equal to the order of an invariant operation of its central quotient group can be written in monomial form.

(4) Fite, loc. cit. vol. 3 (1902) p. 351.

(5) Blichfeldt, Finite collineation groups, p. 79.

$\left.{ }^{8}\right)$ Cf. Blichfeldt, Trans. Amer. Math. Soc. vol. 5 (1904) pp. 313-314; vol. 6 (1905) p. 232. 
This theorem can be stated in the following alternative form:

THEOREM IV'. Let $T$ be a substitution of an irreducible group $G$ that corresponds to an invariant operation of the central quotient group of $G$. Then if the substitutions of $G$ that are commutative with $T$ form an abelian group, $G$ can be written in monomial form.

If the central of $G_{1}$ is not cyclic the irreducible components of $G_{1}$ are not simply isomorphic with it. If, on the other hand, these components are not simply isomorphic with $G_{1}$ there is a substitution of $G_{1}$ all of whose multipliers in one of these components, say $G_{1,1}$, are equal to 1 . Let $T$ be such a substitution. If it is not invariant in $G_{1}$ let $S_{1}$ be a substitution of $G_{1}$ that is not commutative with $T$; say

$$
S_{1}^{-1} T S_{1}=T T_{1}
$$

The multipliers of $T_{1}$ in $G_{1,1}$ are all equal to 1 . If $T_{1}$ is not invariant in $G_{1}$ there is a substitution $S_{2}$ of $G_{1}$ such that

$$
S_{2}^{-1} T_{1} S_{2}=T_{1} T_{2}
$$

where $T_{2}$ is not the identity, but has all its multipliers in $G_{1,1}$ equal to 1 . If it is not invariant in $G_{1}$ and $G_{1}$ is of order $p^{m}$ we can continue this process until we arrive at a $T_{i}$ which is a nonidentity invariant substitution of $G_{1}$ all of whose multipliers in $G_{1,1}$ are equal to 1 . If now $G_{1}$ had a cyclic central generated by $R$ then $R$ and $R T_{i}$ would be two distinct invariant substitutions of $G_{1}$ of the highest order which are identical in $G_{1,1}$. Then $R T_{i}$ is a power of $R$, say $R T_{i}=R^{x}$, where $x \neq \equiv 0(\bmod p)$. Since $R$ and $R^{x}$ are the same as far as concerns the variables of $G_{1,1}$ we have $x \equiv 1\left(\bmod p^{\beta}\right)$, where $p^{\beta}$ is the order of $R$. Hence $R$ and $R T_{i}$ are the same throughout and $T_{i}=1$. But this is contrary to our supposition regarding $T_{i}$. Hence we have:

THEOREM V. If $G$ is of order $p^{m}$ a necessary and sufficient condition that the irreducible components of $G_{1}$ be simply isomorphic with it is that its central be cyclic.

Suppose now that $G$ is irreducible of order $p^{m}$ and degree $p$. We assume further that it is of class $k>2$. It has only $p$ invariant commutators, since the number of these does not exceed the degree of the group (Theorem I). Since the degree of each of the irreducible components of $G_{1}$ is a power of $p$, this degree is 1 and $G_{1}$ is abelian. Moreover the central of $G^{\prime}$ is of order $p$. This can be seen as follows: $\beta=1$, since no invariant operation of $G^{\prime}$ is of order greater than $p$. Suppose now that $S$ and $T$ corresponded to independent invariant operations of $G^{\prime}$. If they were not commutative $G^{\prime}$ would be abelian, and if they were commutative and $A$ were a substitution of $G$ not in $G_{1}$, we should have 


$$
A^{-1} T A=T T_{1} \text { and } A^{-1} S A=S T_{1}^{x},
$$

where $T_{1}$ is invariant in $G$. Then $T^{-x} S$ would be invariant in $G$. But this contradicts the hypothesis in regard to $S$ and $T$. Then $G^{\prime}$ has a central of order $p$ and an abelian subgroup of index $p$, namely, the subgroup that corresponds to $G_{1}$. Then $G^{\prime \prime}$ is either abelian or has a central of order $p$. It follows in the same way that each of the successive central quotient groups, except the last one, has a central of order $p$ : And the last one is of order $p^{2}$, since $G_{1}$ is abelian ( $\left.{ }^{7}\right)$. The commutator subgroup $K$ of $G$ is abelian and of order $p^{m-\alpha-1}$, and $G$ is of $\operatorname{class}\left({ }^{8}\right) k$, where $k=m-\alpha$.

Let $G$ be an irreducible group of order $p^{m}$ and degree $p^{\beta}$ whose central quotient group has an invariant operation of order $p^{\beta}$, and suppose that the commutator subgroup $K$ is generated by a substitution $T_{1}$ of order $p^{\gamma}$. Then there is a substitution $A$ of $G$ and a substitution $T$ of $G_{1}$ such that

$$
A^{-1} T A=T T_{1} \text {. }
$$

For there are two substitutions, $B$ and $C$, of $G$ such that

$$
C^{-1} B^{-1} C B=T_{2},
$$

where $T_{2}$ is of order $p^{\gamma}$. Then there are two substitutions, $B_{1}$ and $C_{1}$, of $G_{1}$ such that $B=B_{1} A_{1}^{b}$ and $C=C_{1} A_{1}^{c}$, where $A_{1}$ together with $G_{1}$ generates $G$. Then

$$
T_{2}=A_{1}^{-c} C_{1}^{-1} A_{1}^{-b} B_{1}^{-1} C_{1} A_{1}^{c} B_{1} A_{1}^{b} \text {. }
$$

From this it is clear that $T_{2}$ is the product of commutators formed by certain powers of $A_{1}$ and certain substitutions of $G_{1}$. Among these commutators there is one of order $p^{\gamma}$. There is then in $G_{1}$ a substitution $T$ and in $G$ a substitution $A$ such that

$$
A^{-1} T A=T T_{1}
$$

where $T_{1}$ is of order $p^{\gamma}$. Then, if $p$ is an odd prime,

$$
A^{-p^{\beta}} T A^{p^{\beta}}=T T_{1}^{p^{\beta}(1+x p)}=T,
$$

since $G_{1}$ is abelian and $A_{1}^{p \beta}$ is in $G_{1}$. This requires that $T_{1}^{p \beta}=1$ and therefore that $T_{1}$ be invariant in $G$. Hence $G$ is metabelian. Moreover $G$ is of order $p^{\alpha+2 \beta}$ and its central quotient group has two independent generators each of order $p^{\beta}$.

THEOREM VI. If an irreducible group $G$ of order $p^{m}$ ( $p$ an odd prime) and degree $p^{\beta}$ has a cyclic commutator subgroup and a central quotient group with an invariant operation of order $p^{\beta}, G$ is metabelian and $m=\alpha+2 \beta$.

(7) Cf. Fite, loc. cit. vol. 3 (1902) p. 342.

(8) Cf. Fite, loc. cit. vol. 7 (1906) p. 68. 
If $G$ is of degree $p$ there are only $p$ invariant commutators, and if $K$ were cyclic of order greater than $p$ there would be a commutator of order $p^{2}$ that corresponds to an invariant operation of $G^{\prime}$. But this is impossible (Theorem II). Hence $K$ is of order $p$, and $G$ is metabelian. This is a special case of the following theorem:

THEOREM VII. If $G$ is an irreducible group of order $p^{m}$ ( $p$ an odd prime) and degree $p^{n}$ with a cyclic commutator subgroup of order $p^{\gamma}$, then $\gamma \leqq n$ and $k \leqq \gamma-\beta+2$.

To see this consider that $H_{2}$ is not $\operatorname{cyclic}\left({ }^{9}\right)$ and let $T$ be a substitution of $H_{2}$ of order $p$ that is not in $H_{1}$. Then $T$ has $p$ conjugates and is invariant in a reducible $G_{2}$ of order $p^{m-1}$. The irreducible components of $G_{2}$ are of degree $p^{n-1}$ and have cyclic commutator subgroups of order $p^{\gamma_{1}}$, where $\gamma-1 \leqq \gamma_{1} \leqq \gamma$. If we look upon one of these irreducible components just as we have looked upon $G$ and continue in this way we would come to an irreducible group of degree $p$ with a cyclic commutator subgroup of order greater than $p$ if $\gamma$ were greater than $n$, since as we proceed the order of the respective commutator subgroups does not decrease more rapidly than the degree of the groups. If $G$ contains $p^{\beta}$ invariant commutators then $\gamma \geqq \beta+k-2$, or $k \leqq \gamma-\beta+2$.

COROLlARY. If a group $G$ of order $p^{m}$ ( $p$ an odd prime) has a cyclic central and a cyclic commutator subgroup of order greater than $p$ it does not contain an abelian subgroup of order $p^{m-1}$.

For if it did it would be simply isomorphic with an irreducible group of degree $p$.

If $p^{\beta_{1}}$ is the highest order of any invariant commutator of the central quotient group and $k>2$ then $\gamma \geqq 2 \beta_{1}$, and therefore $n \geqq 2 \beta_{1}$. That is, if $n<2 \beta_{1}$, $G$ is metabelian. More generally, if $p^{\beta_{1}}$ is the highest order of any commutator of $G / H_{k-2}$ then $\gamma \geqq(k-1) \beta_{1}$ and therefore $n \geqq(k-1) \beta_{1}$.

TheоReм VIII. If the irreducible group $G$ of order $p^{m}$ ( $p$ an odd prime) has a cyclic commutator subgroup and is of degree $p^{\beta+1}$ then $G_{1}$ is metabelian.

For if the commutator subgroup of $G$ is cyclic, that of $G_{1}$ and those of its irreducible components are cyclic. Moreover each of these components is of degree $p$. Hence its commutator subgroup is of order $p$ and it is metabelian. Hence $G_{1}$ is metabelian.

( ${ }^{\circ}$ Fite, loc. cit. vol. 15 (1914) p. 48.

Rollins College,

Winter Park, Fla. 